\title{
Measurement of kernel swelling and buffer densification in irradiated UCO-TRISO particles
}

\author{
Gordon R. Bower ${ }^{\text {a }}$, Scott A. Ploger ${ }^{\text {a, Paul A. Demkowicz }}{ }^{\text {a }}$, John D. Hunn ${ }^{\text {b }}$
}

Keywords: TRISO, fuel swelling, coated particle fuel, post-irradiation examination, ceramography, maximum likelihood estimation, measurement uncertainty

\begin{abstract}
Radiation-induced volume changes in the fuel kernels and buffer layers of UCO-TRISO particles irradiated to an average burnup of 16.1\% FIMA have been determined. Measurements of particle dimensions were made on polished cross-sections of 56 irradiated particles at several different polish planes. The data were then analyzed to compute the equivalent spherical diameters of the kernels and the various coating layers, and these were compared to the average as-fabricated values to determine changes due to irradiation. The kernel volume was found to have increased by an average of $26 \pm 6 \%$. Buffer volume decreased by an average of $39 \pm 2 \%$ due to densification.
\end{abstract}

1.

\section{1.}

The Advanced Gas Reactor (AGR) Fuel Development and Qualification Program was established to perform the requisite research and development on tristructural isotropic (TRISO)-coated particle fuel to support deployment of a very high temperature reactor [1]. Fuel irradiation experiments are being conducted at the Idaho National Laboratory (INL) to obtain data on fuel performance under irradiation, support fuel process development, qualify the fuel for normal operating conditions, provide irradiated fuel for accident testing, and support the development of fuel performance and fission product transport models. The first of these irradiation tests, designated AGR1, ended in November 2009. The experiment completed 620 effective full power days of irradiation in the Advanced Test Reactor. A peak compactaverage burnup of $19.6 \%$ fissions per initial metal atom (FIMA) was achieved with zero particle failures observed based on the measured fission gas release-to-birth rate ratios [2,3].

The AGR1 fuel particles and compacts were fabricated at Oak Ridge National Laboratory (ORNL) using $350 \mu$ mdiameter mixed uranium oxide/uranium carbide (UCO) kernels produced by BWXT Nuclear Operations Group, Inc. [4-6]. All kernels came from a single composite lot with a uranium enrichment of $19.7 \%$ [7]. The kernels were coated sequentially with a $100 \mu \mathrm{mthick}$ porous carbon buffer, a $40 \mu \mathrm{m}$ inner pyrolytic carbon (IPyC) layer, a $35 \mu \mathrm{m}$ silicon carbide (SiC) layer, and a $40 \mu \mathrm{m}$ outer pyrolytic carbon $(\mathrm{OPyC})$ layer. The average envelope density of the buffer layer was $1.10 \mathrm{~g} \mathrm{~cm}^{3}$. As shown in Figure 1 , the particles were pressed into right cylindrical compacts that were nominally $25 \mathrm{~mm}$ in length and $12.4 \mathrm{~mm}$ in diameter, and each compact contained approximately 4,100 coated particles. The UCO kernels can be distinguished easily in the xradiograph, along with the $\sim 1.5 \mathrm{~mm}$ thick fuel-free end caps at the top and bottom. A total of 72 fuel compacts were irradiated in the AGR1 experiment. 

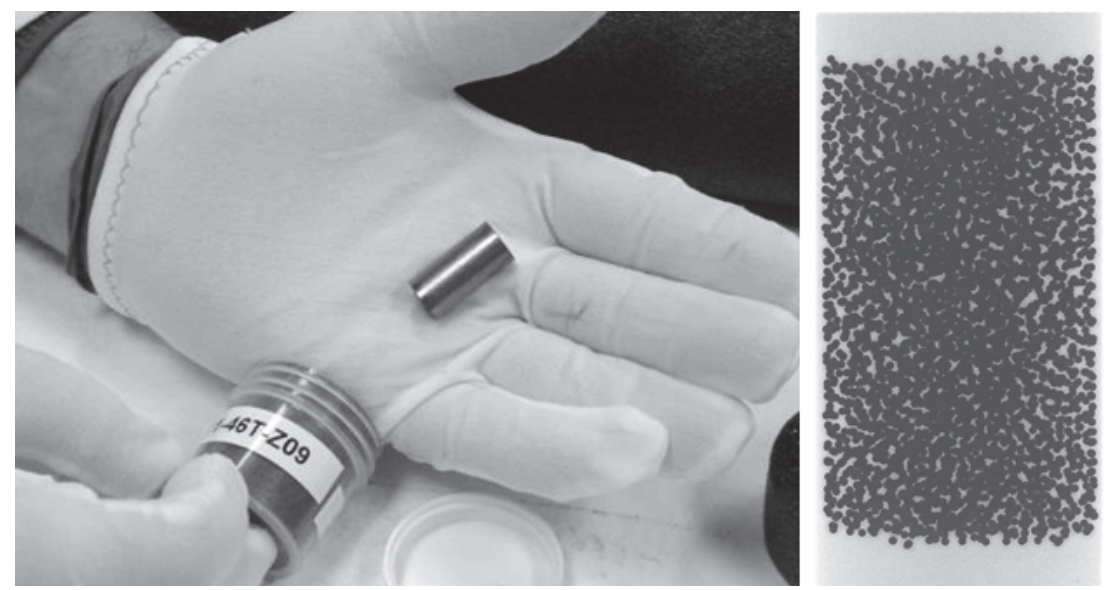

Figure 1. Photograph (left) and x-radiograph (right) of an unirradiated AGR-1 fuel compact.

Post-irradiation examination (PIE) of the AGR-1 fuel was performed at INL and ORNL to assess irradiation performance. A key component of this work involved microanalysis of the fuel particles to examine radiation-induced changes in the kernel and coating microstructures, the extent of coating damage, and fission product migration in the coating layers. Among the data of interest in these examinations was the amount of UCO kernel swelling and buffer shrinkage due to radiation-induced densification. The fuel kernels swell during irradiation due to the generation of fission gas bubbles and incorporation of fission products into the microstructure. Data on the swelling of UCO fuel is particularly scarce in the technical literature, as UCO kernels have been historically utilized much less compared to other kernel compositions, including $\mathrm{UO}_{2}, \mathrm{UC}_{2}$, and $\mathrm{ThO}_{2}$. Buffer densification is mostly driven by neutron damage to the pyrocarbon microstructure. The densification results in a reduction in the buffer layer thickness, and is often accompanied by the buffer pulling away from the IPyC, creating a gap between the two layers. Reliable data on the rates of these phenomena are important for TRISO fuel performance models.

Optical microscopy (ceramography) was performed on cross-sections of AGR-1 fuel compacts [8,9]. While the results yielded a large volume of information on the behavior of particles under irradiation, it was found that extraction of quantitative data on the dimensional changes of the kernels and buffers was problematic for a variety of reasons, as summarized below. As a result, the analysis of loose particle arrays was pursued, and the results are presented in this paper.

\subsection{Ceramography of AGR1 Compacts}

Six irradiated AGR-1 compacts were chosen to span the ranges of irradiation temperature and neutron exposure in the AGR-1 experiment. These compacts were cross-sectioned to investigate visuallyconspicuous phenomena such as morphological changes inside the TRISO particles. This effort provided clear evidence of kernel swelling and buffer densification during irradiation [8]. For example, the kernel often protruded into openings when the buffer layer fractured, as displayed in Figure 2. In this situation, kernel swelling probably helped widen the split between the buffer segment partially bonded to the IPyC layer and the buffer segment that appears completely delaminated from the IPyC in this plane of observation. Less than one-fourth of the characterized particles revealed fractured buffers in the plane of polish, however. More typically, as shown in Figure 3, the buffer appeared to be completely delaminated along the IPyC interface and shrank radially inward to open a substantial gap between the buffer and IPyC layers. In particles with extensive buffer-IPyC delamination, the kernel and surrounding buffer were often offset from center, and were sometimes free to move within the particle during grinding until the radial gap was impregnated with epoxy under vacuum during sample preparation, so the kernel and buffer were often not concentric with the outer three TRISO layers in the final polished cross section. 


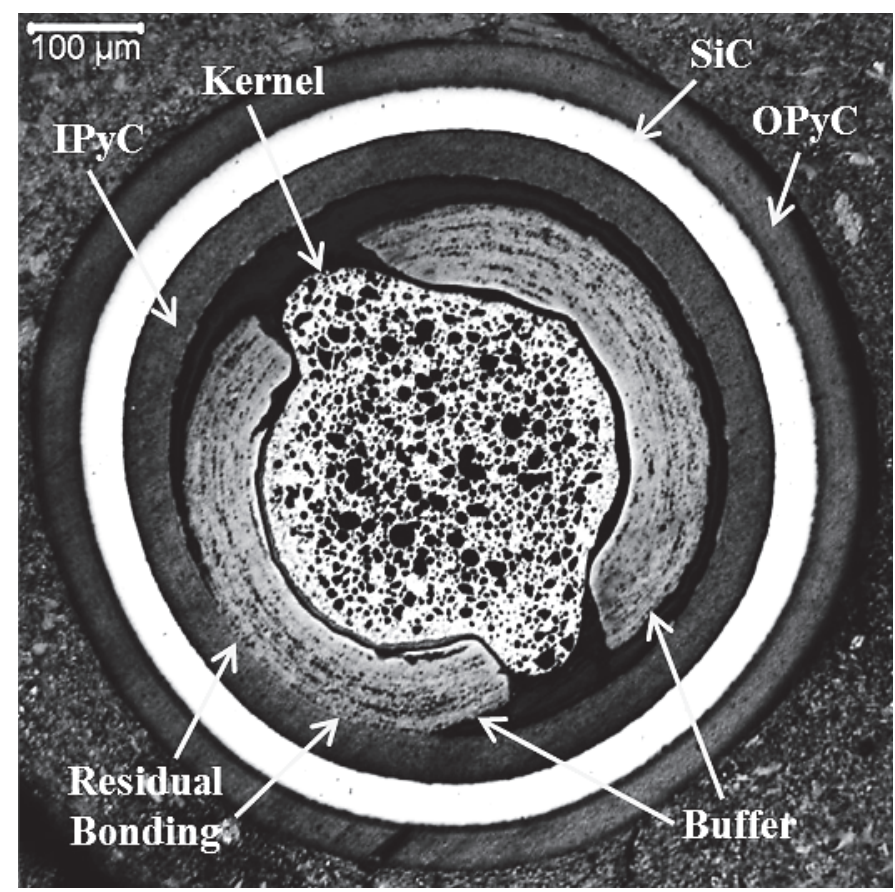

Figure 2. Irradiated AGR-1 particle with kernel protrusions into buffer fractures.

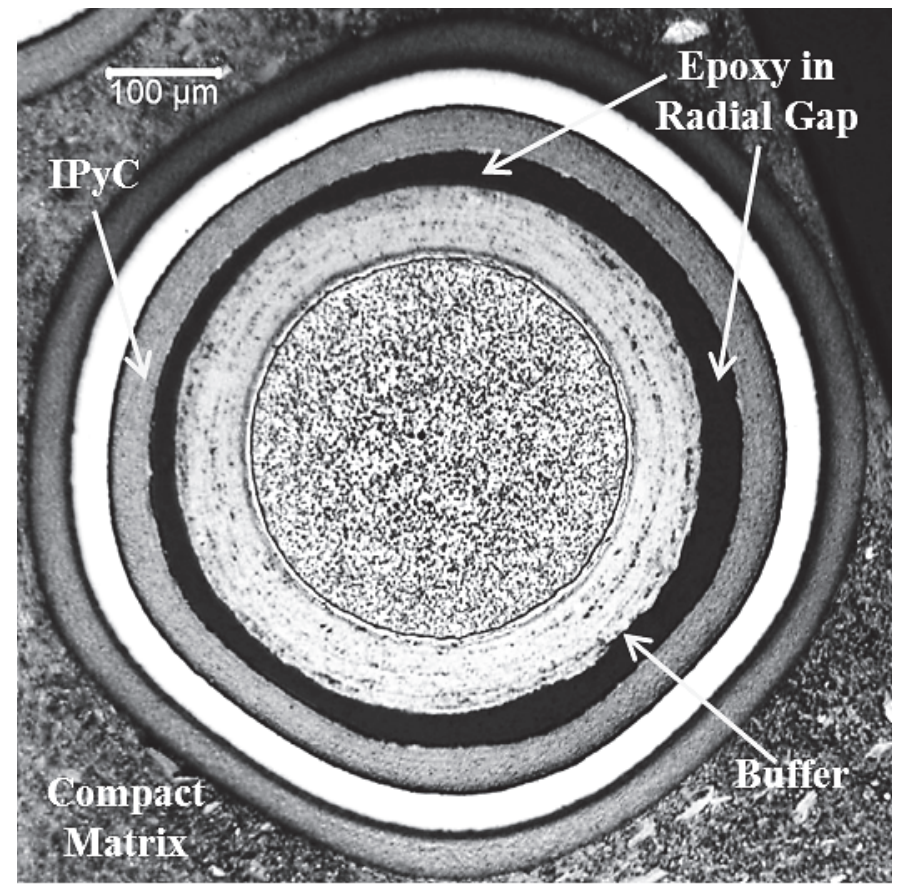

Figure 3. Radial gap commonly formed between buffer and IPyC layers from buffer densification.

The size of the radial buffer-IPyC gap is of special interest for modeling heat transfer from the kernel to the compact matrix [10]. Gap size is predominantly determined by kernel swelling and buffer densification because dimensions of the outer three TRISO layers change relatively little during irradiation. Accordingly, an attempt was made to measure both kernel and buffer areas on particle images from transverse cross sections through three representative compacts, as well as to measure the average buffer-IPyC gap thicknesses. This effort proved largely unsuccessful because the resulting data exhibited 
very wide distributions (some of which were caused by substantial variations in as-fabricated kernel sizes and TRISO layer thicknesses) [10]. Other major factors complicating the analysis of particle dimensions based on measurements made in a single cross-section are summarized below.

- Particles sectioned significantly away from the midplane were difficult to analyze, since the measurements of apparent particle radii in the plane of polish differed significantly from the true spherical radii. The latter can be directly measured only in a midplane cross section, and converting an apparent radius to a true spherical radius requires knowledge of the vertical distance from the midplane.

- In cases where the buffer delaminated from the IPyC, the kernel and surrounding buffer were not concentric with the outer layers. The resulting lack of concentricity made it impossible to directly determine gap widths and distorted kernel and buffer sizes from a single cross section.

- Fractured buffers and protruding kernels could deviate strongly from a spherical geometry, but these features were not necessarily obvious when only a single plane was exposed.

It was therefore recognized that a different approach would be needed to measure the dimensions of the irradiated particles and to subsequently determine the magnitude of the changes relative to average asfabricated dimensions.

\section{Ceramography of Deconsolidated AGR-1 Particles}

Consideration of the difficulties enumerated above created the concept of cross-sectioning a planar array of many particles at multiple elevations, followed by fitting spherical surfaces to the circular radii measured in each plane, to estimate the spherical radii of each particle. In principle, such an approach offered several potentially important advantages:

- Placing loose particles at the same elevation would eliminate the staggered particle elevations in cross-sectioned compacts, thereby enabling most particles in a mount to be fully characterized with a realistically-small number of polished planes.

- Using data from multiple levels to establish kernel and buffer radii diminishes the impact of errors in any one observation, enabling a more precise estimation of radii, and, in turn, estimation of irradiated kernel and buffer volumes.

- Measurements on kernels and buffers could be decoupled from outer TRISO shells, so internal concentricity would not need to be assumed and error from an uneven radial gap could be mitigated.

- Mounting particles in translucent epoxy (as opposed to an opaque compact matrix) would permit a silhouette measurement of the outer radius of each particle.

- Comparing outer particle radii in polished planes to silhouette radii would allow determining distances between levels on an individual particle basis.

These potential advantages could not be realized without substantial development of methods for sample preparation, dimensional measurement, and data analysis. All aspects had to be confirmed in practice on a single metallographic mount in a proof-of-principle demonstration before any consideration could be given to a larger effort.

\subsection{Mounting, Grinding, and Polishing}

Numerous AGR1 fuel compacts were deconsolidated as part of the PIE campaign [11]. Particles from Compact 131 were selected for this analysis. ${ }^{1}$ This fuel specimen had a compact-average burnup of $16.1 \%$ FIMA, a fast neutron fluence of $3.22 \times 10^{25} \mathrm{n} \mathrm{m}^{2}(\mathrm{E}>0.18 \mathrm{MeV})$, and a time-average, volume-average irradiation temperature of $1092^{\circ} \mathrm{C}$ [2]. The particles used in this study had the $\mathrm{OPyC}$ removed during the

\footnotetext{
${ }^{1}$ The AGR-1 fuel compact naming convention refers to the specimen's original location in the irradiation experiment, as described in Reference 11.
} 
leach-burn-leach procedure [11]. A total of 99 randomly selected particles were mounted for analysis, which provided an adequately-large sample for comparing results to as-fabricated averages.

Several mount designs had been tested previously for evenly securing a large ensemble of particles in epoxy. The iteration ultimately implemented for this proof-of-principle demonstration is shown in Figure 4. A thick piece of glass-backed, double-sided tape (not shown) was first attached to the base of a plastic laminate mount. The polycarbonate pedestal was then pressed into the tape at the center of the opening to later force particles away from the mount center. Ten zirconia spheres were then manually pressed into the tape as potential aids for checking grinding depth (not used in actual practice). After this assembly was inserted in a hot cell, the irradiated particles were poured through a funnel and distributed around the annulus by the pedestal. Tape adhesion prevented particles from floating upward as a two-part epoxy was gradually added to fill the annulus. Most of the air trapped as bubbles between particles was removed by applying a vacuum. The vacuum also removed air trapped in the annular gaps, which locked the epoxy to both the mount and the pedestal as it was cured in a furnace. The epoxy shrank somewhat during curing, forming a conspicuous meniscus at the mount top. A slight meniscus also formed at the base, despite the glass-backed double-sided tape. However, locating the particles toward the outside of the mount opening kept most particles close to co-planar during curing.

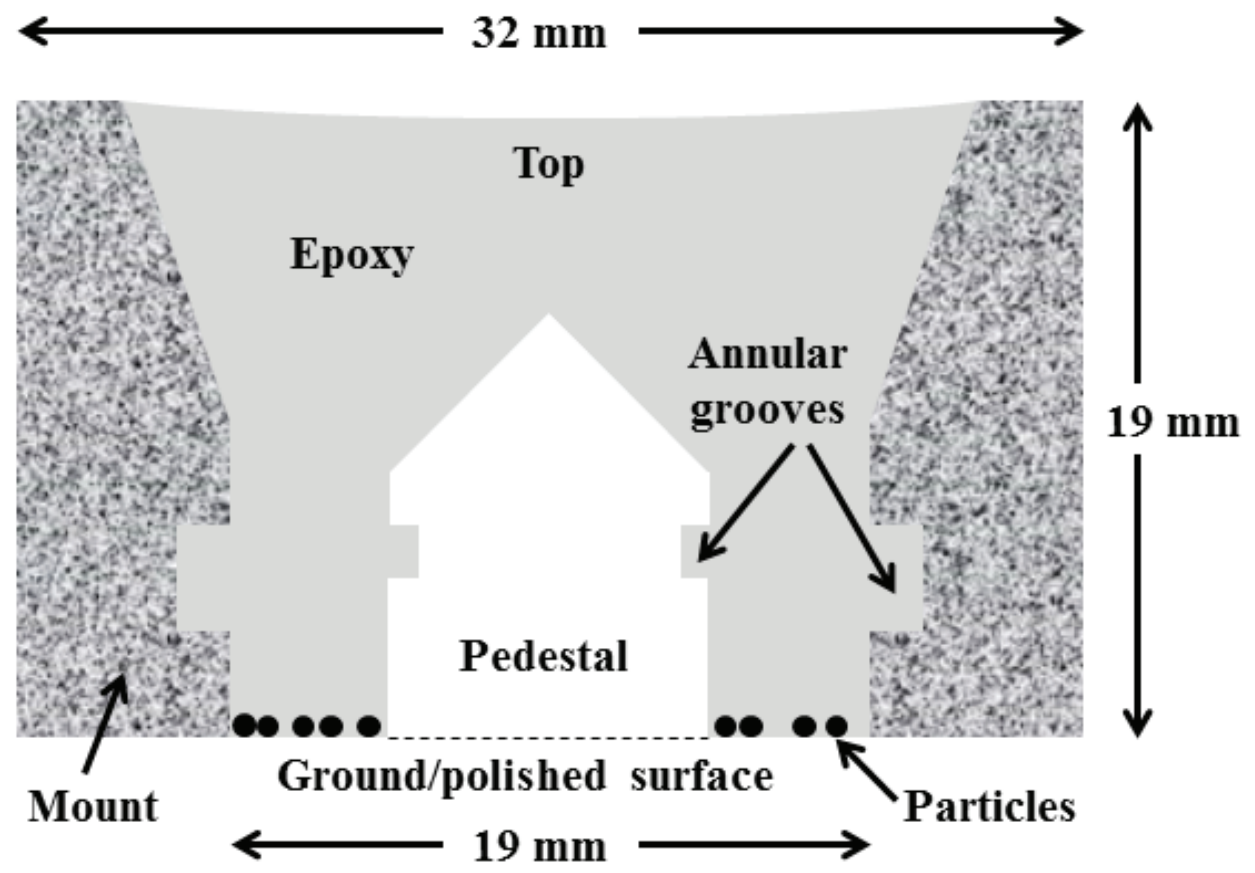

Figure 4. Cross section of mount design developed for large planar arrays of irradiated AGR-1 particles.

The cured mount was transferred pneumatically to a different facility for grinding, polishing, and ceramography. The glass and tape were pried off upon arrival, followed by grinding with a $1200 \mathrm{grit}$ disc until buffer-IPyC gaps could be seen through the periscope in some of the particles. The mount was then vacuum-impregnated with a thin layer of epoxy to stabilize buffers in the particles with open gaps. Grinding continued until buffer-IPyC gaps were apparent in virtually all particles. The mount was then vacuum-impregnated with epoxy for a second time to fill the recently opened gaps and lock the buffers and enclosed kernels in place.

Grinding continued with minimal force until kernels could be seen in the vast majority of the particles. The mount was then briefly polished with a $3 \mu \mathrm{m}$ diamond suspension on a Struers MD-Mol cloth, which adequately defined coating layer edges without prohibitively rounding them.

Low magnification images were taken across the mount on a shielded metallograph and assembled into a montage (Figure 5). After assigning a numeric identifier to each particle, images were taken of 
individual particles at the largest available magnification that would capture each one within the field of view. A silhouette image of each particle was also taken at the same magnification with the aid of a lightemitting-diode flashlight and a diffuser plate, both of which rested inside a fixture on the mount top to backlight the particles.

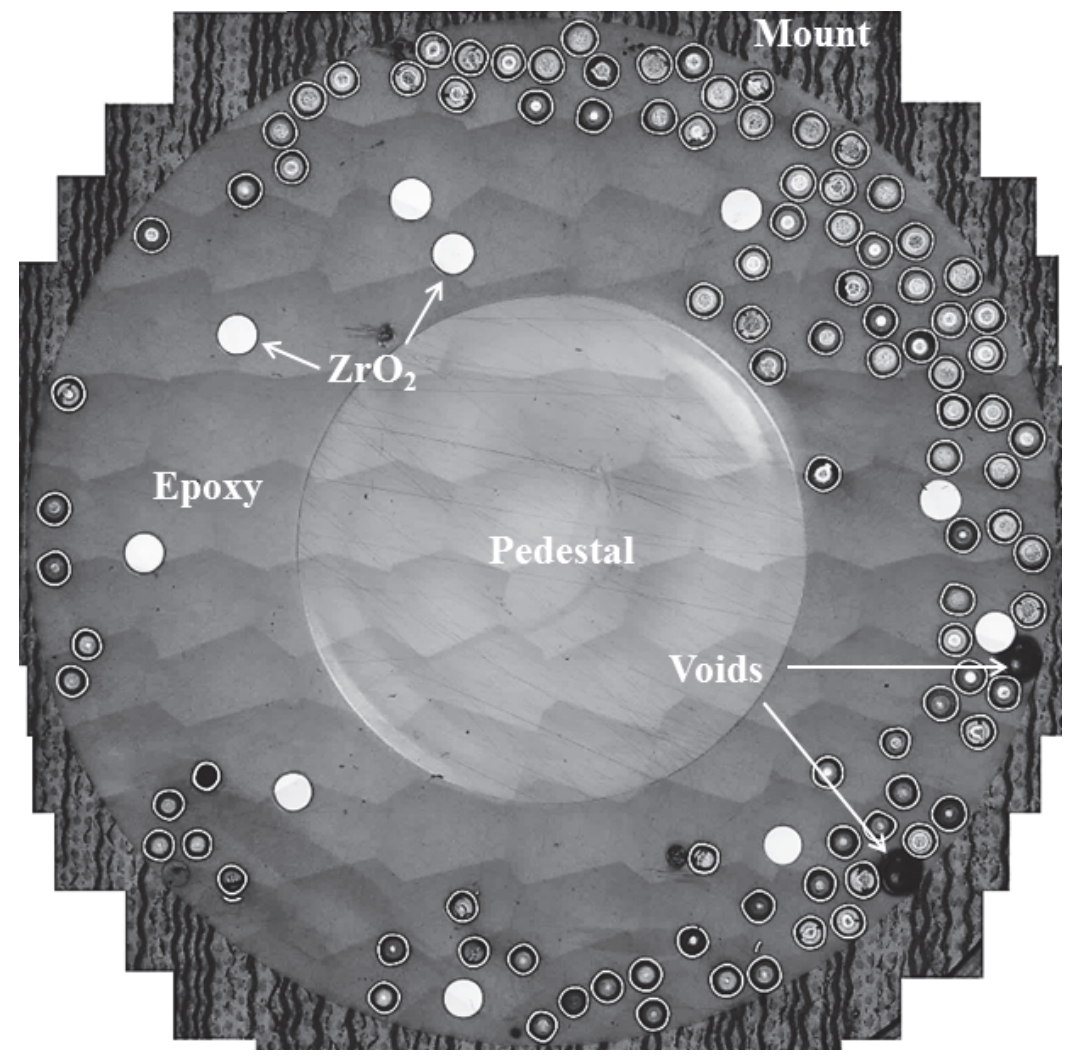

Figure 5. Montage of the first polished level.

Three more levels were ground, polished, and examined in much the same fashion. Distances between levels were coarsely controlled with the aid of a dial indicator-based height gauge that made its measurements close to the mount center on the pedestal. (The analysis discussed in Section 3 found considerable variability in amounts of material ground off individual particles between levels, possibly from uneven grinding pressure due to how the pneumatic pushrod on the Struers MD system contacted the upper mount meniscus.) The $\mathrm{SiC}$ layer in the second imaged plane in Figure 6 is not as well polished as the other planes because a stiffer polishing cloth and larger force were inadvertently used for the final polish; this did not noticeably compromise identification of layer edges. As indicated in Figure 6, the four levels were constrained within the upper kernel halves, which allowed buffers to be laterally supported by back-potted epoxy. This approach limited buffer/kernel pullout during grinding and polishing to only one of 99 particles. 


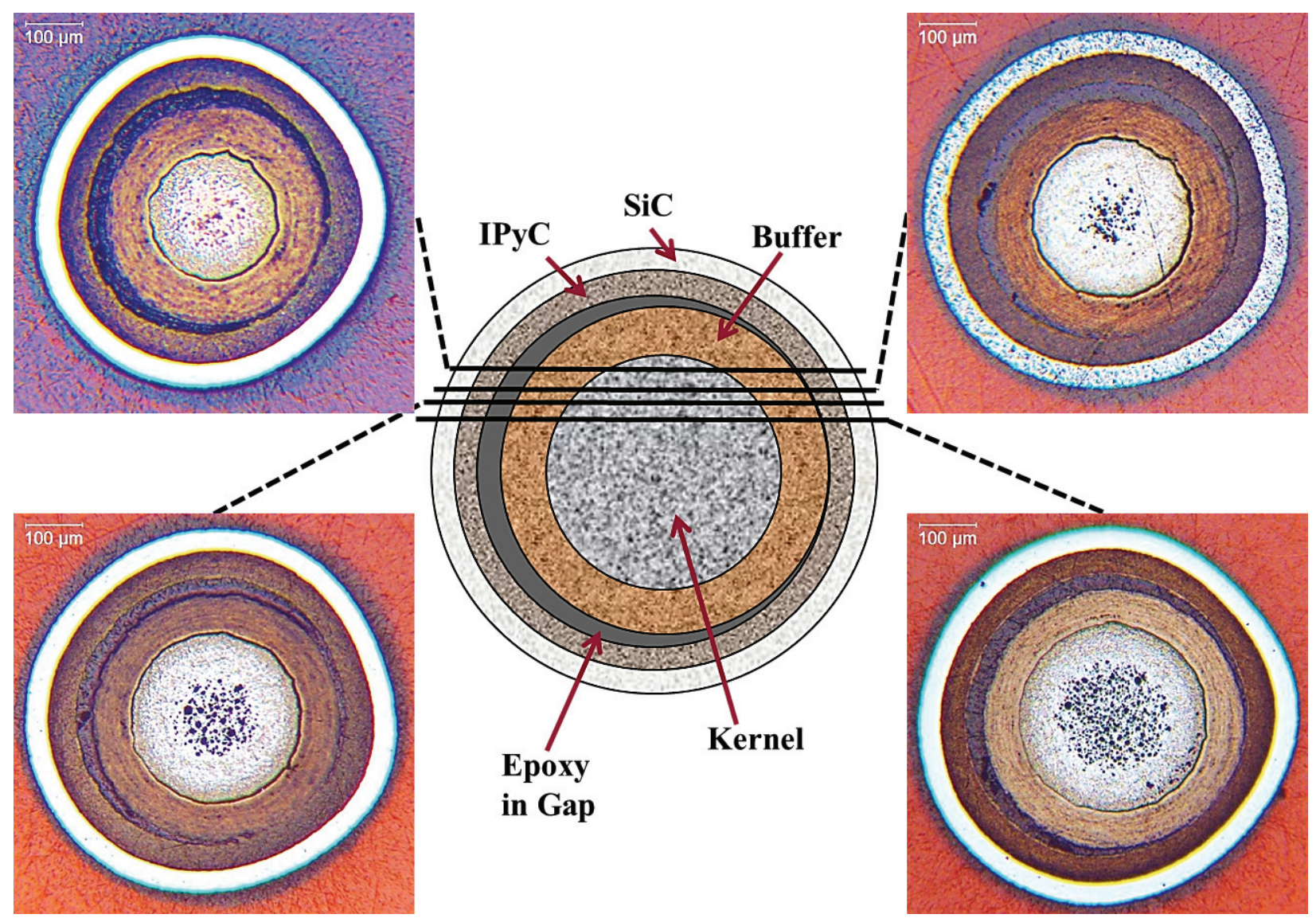

Figure 6. Images of one particle at all four levels examined along with approximate positions of these cross sections within a depth diagram.

\section{2.}

\section{Measurements on Images}

The Figure 6 geometry, with all four levels including a portion of the kernel cross section but still above the kernel midplane, was achieved for 81 of the 98 intact particles. Although 81 particles possessed sufficient exposed kernel for measuring the cross-sectional kernel radius in all four imaged planes, 25 of these particles had fractured buffers and protruding kernels (resembling Figure 2) whose irregular shapes did not conform to a spherical geometry. Consequently, only 56 particles were fully suitable for radial measurements to quantify typical kernel swelling and buffer densification.

The radius of the best-fitting circle for each feature boundary was extracted using PAX-it software from Midwest Information Systems, Inc., after calibrating pixel size to an image of a stage micrometer taken at the same magnification. Kernel radii and outer buffer radii were measured on particle cross sections to assess their respective swelling and densification. Inner buffer radii were assumed identical to the measured kernel radii. Inner radii were measured on IPyC layers, so that the buffer-IPyC gap could be characterized via the difference between inner IPyC radius and outer buffer radius (using the method described in Section 4.3). In the absence of the OPyC layers, outer SiC layer radii were measured on both cross-sectional images and particle silhouettes, where the measured silhouette radii provided estimates of the "true" outer SiC radii at midplane. (Silhouettes can be slightly larger because off-midplane asymmetric bulges may contribute to the projected outermost edge.)

The software computed the radius of each feature by fitting a circle to sixteen manually selected points around the feature boundary. An attempt was made to make the point selection process consistent and diminish subjectivity by selecting points along 8 preferred azimuths. The general approach adopted was to first select points at the top, bottom, left side, and right side of each object. Upper-left, upper-right, 
lower-left, and lower-right points were then picked by visually interpolating midway between the first set of four points. The last eight points were obtained by visually interpolating midway between the previous eight points. To eliminate transcription errors, computed radii from each particle were automatically exported onto a spreadsheet template, along with the five analyzed images and their identifiers.

The approach outlined above for selecting the sixteen points could not be followed in all cases, especially on some buffers. It was not unusual for a layer of buffer to intermittently adhere to the IPyC interior after most of the buffer shrank onto the kernel. An extreme example of this behavior is presented in Figure 7, where stringers of buffer also bridge between the IPyC and buffer bulk. Efforts were made to compensate for such separated buffers when picking points along buffer exteriors. One technique was to move the point outward by an amount that visually corresponded to the buffer thickness attached to the IPyC interior directly across the radial gap. In other situations, the preferred azimuths were rotated slightly to capture the full buffer thickness and avoid local regions where the buffer separated. All of these efforts were unavoidably subjective and likely injected more error in fitting circles to buffer exteriors than elsewhere within the particles where point selection was more straightforward.

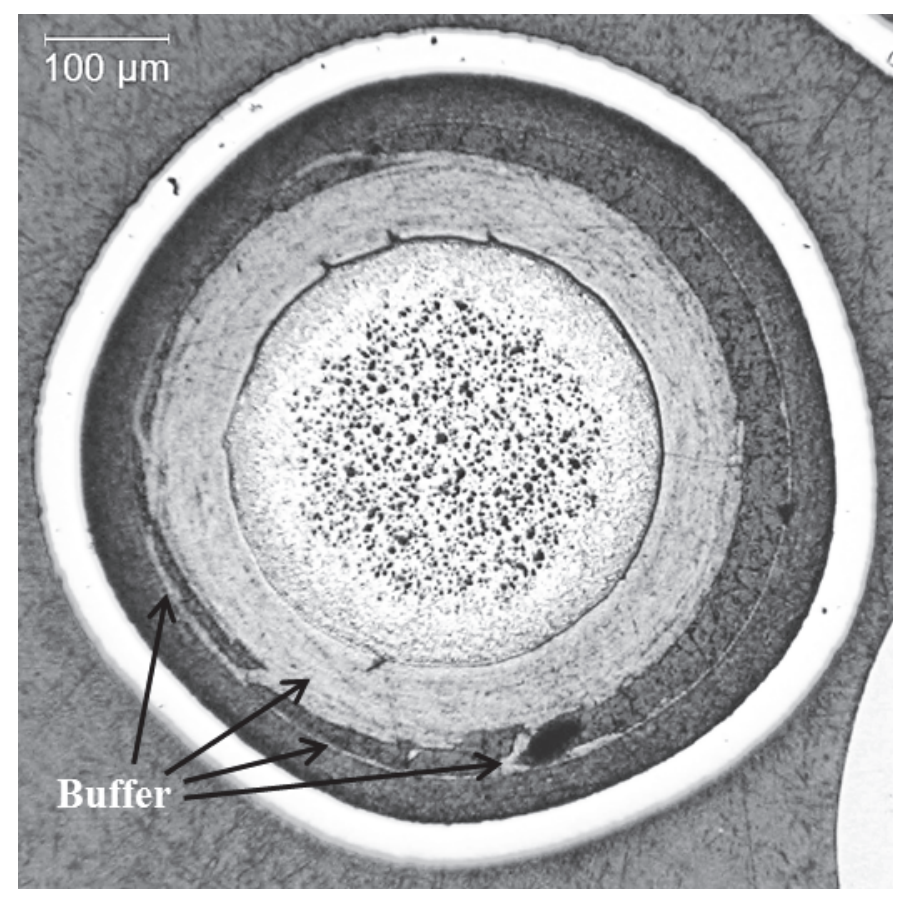

Figure 7. Buffer separation across the buffer-IPyC gap in level 4 of particle 041.

3.

Calculation

\section{1. \\ Description of Mathematical Model}

As described in the previous section, the data collected for further analysis comprised the radii of the best-fit circle for each feature boundary (the outer edge of the SiC, inner edge of IPyC, outer edge of buffer, and the outer edge of kernel), measured in each of four cross sections through the particle, plus one silhouette measurement, which, assuming a spherical particle, served as an additional measurement of the outermost radius at midplane.

The calculation method developed here can handle, in general, measurements of each of $m$ layers in each of $k$ cross-sectional views (and can be adapted to handle cases where not every layer is measured in every cross-section.)

Irradiated particles can be idealized as nested spheres. The outer SiC and IPyC layers are concentric, and the inner buffer and kernel are also concentric with each other, but typically are not concentric with 
the outer layers (as they are in as-fabricated particles) because the inner layers have some freedom of movement after the buffer shrinks.

Consider a model spherical particle (Figure 8) with four spherical shell radii $r_{1}, r_{2}, r_{3}$, and $r_{4}\left(r_{1}\right.$ the outer $\mathrm{SiC}$ radius, $r_{2}$ the inner IPyC radius, $r_{3}$ the outer buffer radius and $r_{4}$ the kernel radius) and vertical offset $z_{M}$ between the midplane of the two outer shells and the midplane of the two inner shells. For four cross sections through that particle at heights $z_{1}, z_{2}, z_{3}$, and $z_{4}$ above the midplane of the outer shells, one can calculate the circular radii $x_{i j}$ that will be observed in those cross sections using simple geometry. For the two outer layers,

$$
\text { , for shells } 1 \leq i \leq 2 \text { and slices } 1 \leq j \leq k \text {. }
$$

For the two inner layers,

$$
\text { , for shells } 3 \leq i \leq 4 \text { and } 1 \leq j \leq k \text {, }
$$

The silhouette measurement of the outermost layer, $x_{10}$, is expected to be close to $r_{1}$.

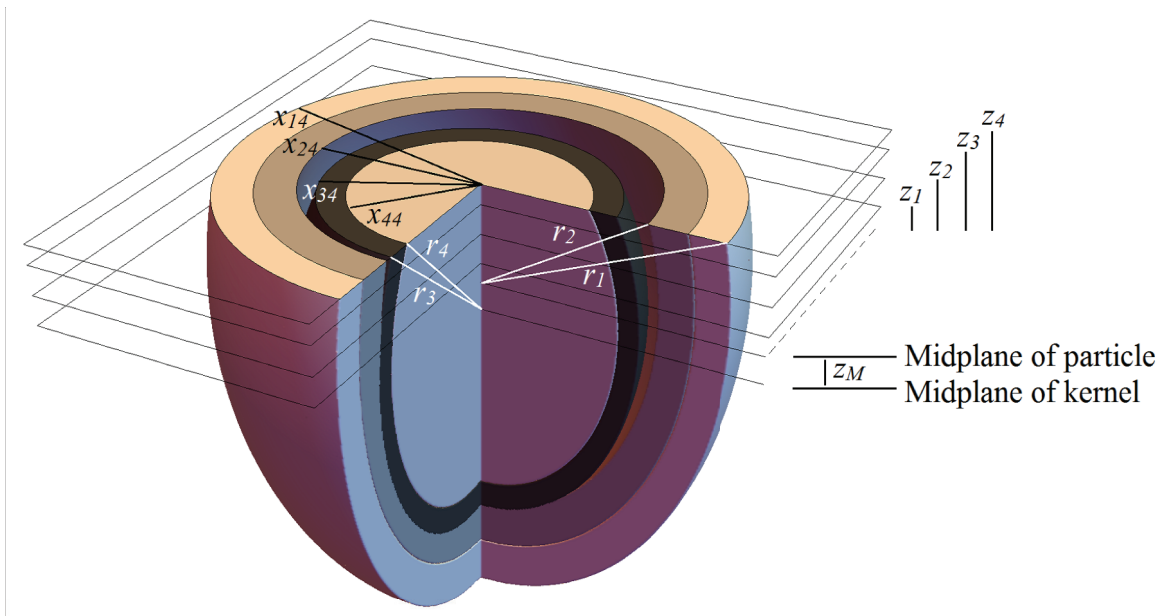

Figure 8. Geometry of an idealized particle. Compare with views of an actual particle shown in Figure 6.

When this method is applied to an actual AGR1 particle, each of the $r_{i}$ and $z_{j}$ are fixed but unknown, and must be calculated from the observations. Meanwhile each of the observations of cross-sectional radii $x_{i j}$ is subject to experimental error. Based on experience with the measurement software and the process described in Section 2.2, including tests where 16 sample points were picked repeatedly from the same image in different orientations and the fitted circular radii compared, the measurement errors appeared to be approximately normally distributed with a standard deviation of about $1 \mu \mathrm{m}$, i.e., the large majority of replicated measurements clustered in a band $\pm 2 \mu \mathrm{m}$ from the mean. The measurements are more stable when the cross-sections are clean, and worse when the particles are highly aspherical, when the crosssection passes almost tangentially across the top of a layer, or when grinding and polishing leaves a rough boundary.

The situation poses a statistical problem, using $m k+1$ observations to estimate $m+k+1$ parameters. (For each of the 56 particles analyzed in this study, $m=4$ and $k=4$, resulting in 17 observations to estimate 9 parameters.)

It would be desirable to directly measure the spacings between cross sections, $z_{1}-z_{2}, z_{2}-z_{3}$, and $z_{3}-z_{4}$, either through very precise control of the grinding process, or by embedding objects of known dimension 
into the mount alongside the fuel particles. This would require the estimation of only $k+2$ parameters and enable more precise determination of the spherical radii for each feature boundary. In practice, conditions on the mount were such that these spacings were known only very approximately (in part because there was substantial variability in local material removal during grinding), and simply using the crosssectional measurements to calculate both the vertical offsets and the particle radii worked adequately.

\subsection{Maximum likelihood fitting}

Maximum likelihood estimation is a widely used general-purpose method of fitting a model to a data set. Briefly, given some assumptions about the nature of the random error present in the data, one determines which values of the model parameters (here, the radii of the 4 spherical surfaces of interest, the vertical displacement of the kernel and buffer relative to the outer layers, and the altitudes of the 4 cross-sections) would most likely have generated the data which were collected (here, the 17 circular radii obtained from the image analysis of the four cross sections). Uncertainty bounds on these parameter values are constructed by observing how much a change in a parameter affects the likelihood of observing the data set.

Given a set of field observations $x_{i j}$ on one particle, and a set of candidate values for the parameter estimates, and, the difference between the observed and expected radii can be computed:

$$
\begin{aligned}
& \text {, for } i=1,2,1 \leq j \leq 4 ; \\
& \text {, for } i=3,4,1 \leq j \leq 4 ; \\
& \text {, for the silhouette measurement. }
\end{aligned}
$$

Assuming the measurement error for each observation to be approximately normal with mean 0 and standard deviation $\sigma_{i j}$, the likelihood function to be maximized is

Equivalently, taking logarithms and dropping constant terms, one seeks to minimize

In the special case where the $\sigma_{i j}$ are equal (as they often will be, when all measurements are taken by the same method), this simplifies to minimizing $\Sigma e_{i j}{ }^{2}$. The maximum likelihood estimate (MLE) is the set of and corresponding to the global minimum of $L$, that is, the usually-unique point at which all of the first partial derivatives of $L$ with respect to each parameter are zero, and all of the second partial derivatives are nonnegative.

All of the equations for the $e_{i j}$ are twice differentiable, so it is easy to calculate the gradient and Hessian (9x9 matrix of second partial derivatives) of $L$ and quickly find a minimum using the method of steepest descent, Newton's method, or a convenient combination of the two. Once the minimum has been located, the variance-covariance matrix of the estimates is obtained by inverting the Hessian of $L$ at the MLE [12]. In addition to reading the uncertainty of each of the $m+k+1$ parameter estimates directly off its main diagonal, the variance-covariance matrix can be used to place error bounds on any linear combination of parameters: for instance, the estimated buffer thickness, , has variance .

An implementation of this algorithm was written in Mathematica, and MLEs were obtained for each of the 56 particles that were deemed suitable for detailed analysis. Summary statistics of these are reported in Section 4 below.

The method also performed satisfactorily on test cases where $\sigma_{i j}$ was not constant (particles where the uppermost cross-section was almost tangential to the kernel, producing a large radius uncertainty on the kernel for that layer only) and on test cases where no silhouette measurement was available, though in the absence of a silhouette measurement, the uncertainty bounds on the estimated spherical radii were typically much wider. 
Even in the presence of a silhouette measurement, there is considerable uncertainty in the estimated kernel and buffer radii. Starting from 17 measurements with $1 \mu \mathrm{m}$ errors, typically the two outer radii could easily be determined within $1 \mu \mathrm{m}$, while the estimated kernel and buffer radii had standard errors in the 1-7 and 2-9 $\mu \mathrm{m}$ range respectively. The altitudes of the cross sections had standard errors of 3-5 $\mu \mathrm{m}$; and the estimated vertical offsets of the kernel relative to the outer shells, standard errors of 5-10 $\mu \mathrm{m}$.

The model-fitting procedure also provides a built-in check as to whether the model assumptions are valid: if the particles truly are spherical shells, and each of the $m k+1$ measurements is independent, and each measurement error is normal with mean 0 and standard deviation $\sigma_{i j}$, then the minimum value of $L$ should be approximately $\chi^{2}$-distributed with $m k-m-k$ (here 8 ) degrees of freedom. Two unusually large values of $L$ were observed among the 56 particles, and these corresponded to two particles that displayed highly non-circular cross sections in the photographs. Across all 56 particles, the average minimum $L$ was 5.24 compared to the expected 8, suggesting that the assumption of $\sigma_{i j}=1$ was in fact somewhat conservative.

\subsection{Calculation of volume changes}

The analysis of Section 3.2 provided an asymptotically-unbiased estimated radius for each kernel, with an associated uncertainty. Simply applying $\mathrm{V}=(4 / 3) \pi r^{3}$ to an estimated radius results in a biased estimate of volume: for instance, a sphere with radius $10 \%$ higher than nominal has a volume $33 \%$ higher, while a sphere with radius $10 \%$ smaller than nominal has a volume only $27 \%$ lower. If $X$ is a normally distributed random variable with mean $\mu$ and variance $\sigma^{2}$, then $\mathrm{E}\left[X^{3}\right]$ is not just $\mu^{3}$, but $\mu^{3}+3 \mu \sigma^{2}$, and $\operatorname{Var}\left[X^{3}\right]=9 \mu^{4} \sigma^{2}+36 \sigma^{4} \mu^{2}+15 \sigma^{6}[13]$. Thus, given an estimated radius $r$, the proper estimate of the enclosed volume is $(4 / 3) \pi\left(r^{3}+3 r \sigma^{2}\right)$.

The average volume of an irradiated kernel can either be calculated by finding an estimated volume for each of the 56 particles and averaging them, or by using the mean kernel radius and its standard deviation in the above formula. For the present study, both methods gave the same answer to four significant digits.

This same method can be applied directly to find the total volume enclosed by any one spherical shell. However, it is usually the volume of one layer (including the "layer" of empty space between the buffer and the IPyC), rather than the total enclosed volume, that is of interest.

As above, a bias correction due to not knowing $r_{3}$ and $r_{4}$ precisely is appropriate in calculating the average buffer volume, as opposed to directly substituting the average inner and outer radii into the equation for buffer volume, $\mathrm{V}=(4 / 3) \pi\left(r_{3}{ }^{3}-r_{4}{ }^{3}\right)$. The unbiased volume estimate is reasonably simple, in terms of $r_{3}, r_{4}$, and their variances $s_{r 3}{ }^{2}$ and $s_{r 4^{2}}:(4 / 3) \pi\left(r_{3}{ }^{3}+3 r_{3} s_{r 3}{ }^{2}-r_{4}{ }^{3}-3 r_{4} s_{r 4^{2}}\right)$. However, the variance of this new estimate does not have a tidy closed form. It requires the covariance of $r_{3}{ }^{3}$ and $r_{4}{ }^{3}$, which is not a simple function of the covariance of $r_{3}$ and $r_{4}$. Therefore average buffer volume was not calculated from the summary statistics. Instead, it was necessary to compute $(4 / 3) \pi\left(r 3^{3}-r 4^{3}\right)$ for each of the 56 particles and calculate the mean and standard deviation (SD) of that list of 56 estimates.

4.

4.1.

\section{Results and Discussion}

\section{Average radii of particles}

The analysis described in Section 3.2 yielded estimates for the outside $\mathrm{SiC}$ radius, inside IPyC radius, outside buffer radius, and kernel radius for each of the 56 particles, as well as estimates for vertical displacement between the center of the kernel and buffer and the center of the outer layers.

Summary statistics are reported in Table 1 . Note that both the uncertainty of the result of each of the 56 one-particle analyses and the variation among particles contribute to the total uncertainty in the mean post-irradiation radii. Variation among particles was the larger contributor. This indicates that, if a more 
precise result is desired from a future experiment, analysis of a larger number of particles, rather than taking more than four cross-sections of each particle, is recommended.

Table 1. Post-irradiation radii of particles

\begin{tabular}{|l|r|r|r|r|}
\hline Estimated post-irradiation radii (microns) & \multicolumn{1}{l|}{$\begin{array}{l}\text { SiC } \\
\text { (outer) }\end{array}$} & $\begin{array}{l}\text { IPyC } \\
\text { (inner) }\end{array}$ & $\begin{array}{l}\text { Buffer } \\
\text { (outer) }\end{array}$ & Kernel \\
\hline Single-particle estimates: minimum & 337.4 & 267.1 & 242.6 & 172.1 \\
\hline median & 354.9 & 283.2 & 257.0 & 189.2 \\
\hline \multicolumn{1}{|c|}{ maximum } & 367.3 & 298.2 & 270.5 & 206.3 \\
\hline & & & & \\
\hline Mean & 354.3 & 282.9 & 256.5 & 189.9 \\
\hline Average SD within a single-particle estimate & 0.7 & 1.0 & 2.9 & 3.9 \\
\hline SD between particles & 6.7 & 7.3 & 6.8 & 8.6 \\
\hline Combined SD & 6.7 & 7.4 & 7.4 & 9.4 \\
\hline & & & & \\
\hline 95\% confidence interval for mean: low & 352.5 & 280.9 & 254.5 & 187.3 \\
\hline high & 356.2 & 284.9 & 258.6 & 192.5 \\
\hline & & & & \\
\hline Average as-fabricated radius [14] & 355.0 & 280.3 & 280.3 & 176.1 \\
\hline As-fabricated SD between particles [14] & 7.6 & 7.8 & 7.8 & 4.6 \\
\hline
\end{tabular}

The data in Table 1 for as-fabricated radii come from pre-irradiation characterization of 240 particles from the TRISO particle composite (LEU01-49T) used to fabricate Compact 1-3-1 [14]. For each particle, the mean kernel radius and mean thickness of each layer were determined from a single cross section using computer-automated image analysis, with a correction factor to account for the polished section not being exactly at midplane, as described in [15]. The average radius of the buffer/IPyC boundary reported in Table 1 is the average of the mean kernel radius plus mean thickness of the buffer layer for each particle (there was no gap in the as-fabricated particles), and the average outer radius of the $\mathrm{SiC}$ comes from adding the thickness of the IPyC and $\mathrm{SiC}$ to this value. The average kernel radius prior to coating was determined by shadow analysis [15] of 4305 kernels from the UCO kernel composite used to make the TRISO particles. The value was $174.8 \mu \mathrm{m}$, with a standard deviation of $4.7 \mu \mathrm{m}$ [14], which is slightly smaller than the value measured by cross sectioning after coating but within the uncertainty of the analysis. These values may also differ slightly due to the different impact of kernel shape on the two analysis methods and a reaction that occurs between the kernel and buffer during coating $[15,16]$. Whereas the shadow imaging measurement of the pre-coated kernels is more often reported for the AGR-1 fuel because it is a specified quality control parameter [7], the value obtained via cross sectioning is more relevant for comparison to data obtained from irradiated particles in this study.

Note from the data in Table 1 that the buffer shrank substantially and the kernel expanded substantially as a result of the irradiation; these results are discussed below. The difference in the outer $\mathrm{SiC}$ radius measured before and after irradiation is within the measurement uncertainty, and the $\mathrm{SiC}$ is not expected to change dimensions by a measureable fraction as a result of the AGR-1 irradiation [17]. The difference in the IPyC inner radius after irradiation could be related to a small fractional change in IPyC dimensions as a result of radiation-induced densification [10], but additional work will be required to 
better quantify this effect and verify the apparent change is not dominated by measurement uncertainty and complications introduced by the buffer/IPyC separation. The position of the SiC-IPyC interface was not measured, because negligible change in the SiC layer was anticipated.

\subsection{Kernel swelling and buffer densification}

As described in Section 3.3, the kernel and buffer radii in Table 1 can be used to calculate the postirradiation volumes of the kernel and the buffer. The mean volume of the irradiated kernels was found to be $0.0289 \pm .0012 \mathrm{~mm}^{3}(95 \%)$. Compared to the nominal as-fabricated mean volume of $0.0229 \mathrm{~mm}^{3}$ [14], a $26 \pm 6 \%$ increase was observed in this experiment. There was considerable variation in individual irradiated kernel volumes, from .021 to $.037 \mathrm{~mm}^{3}$; the observed distribution of irradiated kernel volumes is shown in Figure 9. (Note that Figure 9 stacks the fifty-six uncertainty distributions for each particle, not just their means, so appears smoother than a histogram.)

Available experimental data on the irradiation swelling behavior of UCO fuel is extremely limited. Accordingly, within the US TRISO fuel program, computational modeling typically has used swelling correlations based on $\mathrm{UO}_{2}$ fuel used in light water reactors [18]. The corresponding mean volumetric swelling rate for the Compact 131 irradiation conditions using LWR fuel data is approximately $0.8 \%$ per \%FIMA [19]. While published data on irradiation swelling of TRISO kernels is scarce, Proksch et al. [20] reported values for BISO and TRISO particles with a variety of kernel compositions, kernel diameters, and ${ }^{235} \mathrm{U}$ enrichments. This included results on $400-\mu$ m-diameter $\mathrm{UO}_{2}$ kernels with ${ }^{235} \mathrm{U}$ enrichments of 20 and $40 \%$, irradiated to burnups as high as $16 \%$ FIMA at a reported temperature of $1200^{\circ} \mathrm{C}$. Using the data in Fig. 1 from Ref. 20, average volumetric swelling rates were determined and varied slightly depending on which fuel type (BISO or TRISO) or enrichment is considered. Values range from approximately 1.0 to $1.4 \%$ per $\%$ FIMA for the $20 \%$ and $40 \%$ enrichments (burnup between 3.6 and $16 \%$ FIMA). ${ }^{2}$ The authors also noted that swelling rates appeared to increase with decreasing kernel diameter.

While the average values from Proksch et al. are higher than those based on LWR fuel data, the value reported here for Compact 131 is appreciably higher still (1.6\% per \%FIMA). The reason for this discrepancy is not clear at this time, as fuel swelling can be dependent on a number of fuel properties and irradiation conditions. The difference could be partially attributed to the different kernel composition $\left(\mathrm{UO}_{2}\right.$ vs. UCO). There is also evidence from observation of AGR-1 particles with a fractured buffer and those with a buffer that did not separate from the IPyC layer (resulting in a larger inner radius after radiation-induced densification) that kernel swelling is dramatically impacted by how much the swelling is constrained by the buffer layer $[8,9]$. The implication is that the particles selected for this study (comprising only those particles with intact buffer and buffer-IPyC separation) may have exhibited lower levels of kernel swelling than other particles with fractured buffers or no buffer-IPyC separation, and therefore the average volumetric increase of this population could be biased low. More advanced techniques (such as X-ray tomography of particles) would be needed to provide statistics on postirradiation volumes for kernels with more complex shapes (such as the example in Figure 2) that are not easily fitted to a spherical geometry.

\footnotetext{
2 There was considerable scatter in the data, however, and the swelling rates inferred from single data points could range from 0.9 to $1.6 \%$ per $\%$ FIMA.
} 


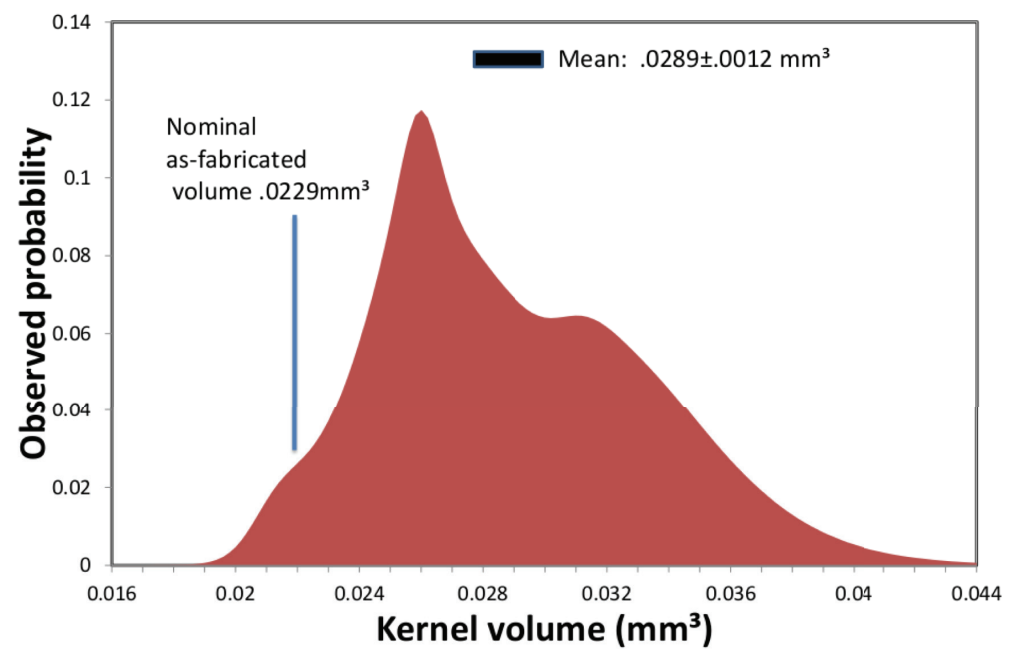

Figure 9. Distribution of estimated volumes of 56 irradiated kernels. Note that because the estimated volume for each kernel had its own mean and standard deviation, this graph is the superposition of those 56 bell-shaped distributions, rather than a simple histogram of the 56 estimated volumes of each particle.

The mean volume of the irradiated buffer was found to be $0.0420 \pm .0013 \mathrm{~mm}^{3}$. Compared to the asfabricated average volume of $0.0694 \mathrm{~mm}^{3}$, this is a $39 \pm 2 \%$ decrease in volume, or, equivalently, a $65 \pm 5 \%$ increase in density. The observed distribution of irradiated buffer volumes is shown in Figure 10.

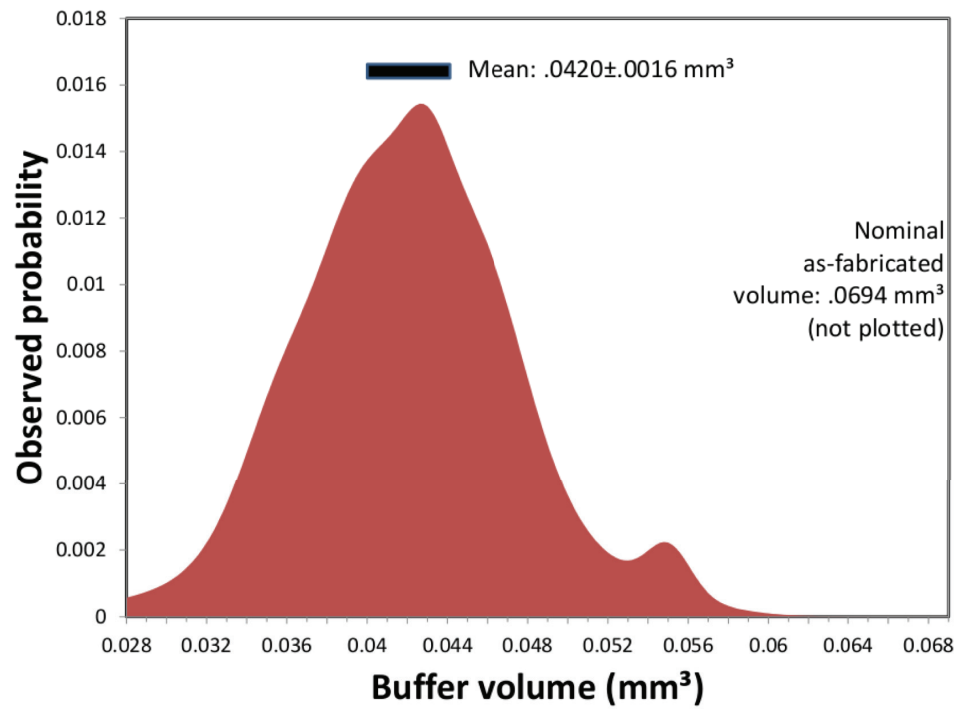

Figure 10. Distribution of estimated buffer volumes in 56 irradiated particles. 


\subsection{Dimensions of gap between buffer and IPyC}

As discussed in Section 1.2, most particles exhibited a gap between the outside of the buffer and the inside of the IPyC layer. Figure 3 is typical of how this gap appeared in a cross sectional view of an irradiated particle.

In AGR-1 fuel, as the buffer shrank, it typically delaminated close to the IPyC interface, with the buffer-coated kernel portion sometimes remaining affixed to the IPyC in one or more locations and sometimes completely detached and free to move about inside the resulting cavity. Typically only a thin residue of the buffer remained attached to the IPyC, but in some cases larger fragments of the buffer were left attached to the IPyC as illustrated in Figure 7, or the buffer layer fractured to expose the kernel as illustrated in Figure 2. Regardless of the extent of buffer-IPyC separation, it is very difficult to estimate the size of the gap by direct examination of the photographs: a cross-section that happens to be taken very near the point where the buffer and IPyC are in contact will appear to show almost no gap while a crosssection on the opposite side of the sphere will show an excessively large gap. Accordingly, $r_{2}-r_{3}$, the difference between inner IPyC radius and outer buffer radius, was used to quantify the gap. The actual gap width displayed in any one cross section may vary from 0 to $2\left(r_{2}-r_{3}\right)$.

The distribution of differences between inner IPyC radius and outer buffer radius is shown in Figure 11. The methods of Section 3.3 could be used to calculate the volume of the void space, but for heat transfer modeling, the average width of the gap is the parameter of greater interest.

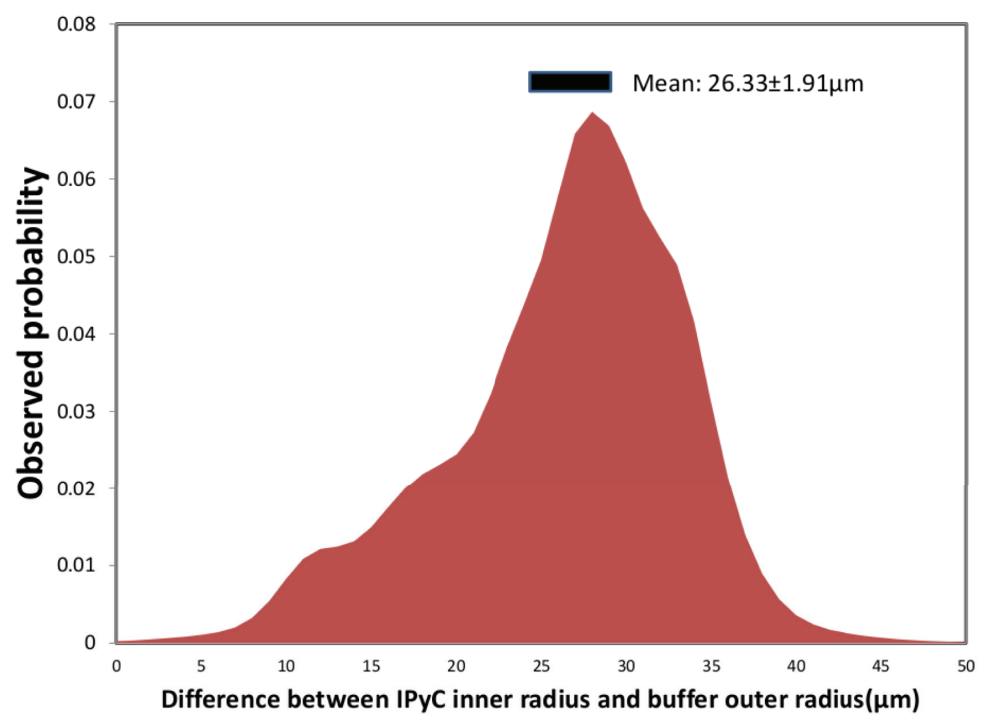

Figure 11. Distribution of buffer-IPyC gap sizes in 56 irradiated particles.

5. Conclusions

An approach for determining the volumes of kernels and coating layers in irradiated TRISO particles has been demonstrated. Because individual as-fabricated particle dimensions vary, and the as-fabricated dimensions for the specific particles analyzed after irradiation were unknown, the post-irradiation average dimensions were compared with similar pre-irradiation averages to compute the average dimensional change for the kernels and the buffer layers. For fuel particles with $350-\mu \mathrm{m}$-diameter kernels, nominal 
burnup of $16.1 \%$ FIMA, and a fast neutron fluence of $3.22 \times 10^{25} \mathrm{n} \cdot \mathrm{m}^{2}$, the results indicate that the mean volume increase in the kernels was $26 \pm 6 \%$ (a net average volumetric swelling rate of $1.6 \%$ per $\% \mathrm{FIMA}$ ) and the mean volume decrease in the buffer layers was $39 \pm 2 \%$. The average difference between the outer radius of the buffer and the inner radius of the IPyC layers in these particles was $26 \mu \mathrm{m}$.

The work presented here was undertaken as a proof-of-principle exercise for determining the extent of radiation-induced dimensional changes in TRISO particles, and has demonstrated the feasibility of the method. Additional measurements may be pursued to explore the correlation of kernel swelling and buffer shrinkage with irradiation conditions (including burnup, neutron fluence, and irradiation temperature), as well as to expand the measurements to include $\mathrm{UO}_{2}$ fuel kernels that are part of the AGR2 irradiation experiment in the U.S.

\section{Acknowledgments}

This work was supported by the U.S. Department of Energy, Office of Nuclear Energy as part of the Advanced Gas Reactor Fuel Development and Qualification Program. The authors would like to thank M.S. Kearns, B.J. Frickey, and C.L. Christensen at the Idaho National Laboratory for preparation of the irradiated particle mount, and G.W. Helmreich at Oak Ridge National Laboratory for assisting with additional quantitative image analysis of some of the historical data from as-fabricated particle cross sections.

\section{References}

1. D.A. Petti, J.T. Maki, J.D. Hunn, P.J. Pappano, C.M. Barnes, J.J. Saurwein, S.G. Nagley, J.M. Kendall, R.R. Hobbins, The DOE advanced gas reactor fuel development and qualification program, JOM 62 (2010) 62-66.

2. B.P. Collin, AGR-1 Irradiation Test Final As-Run Report, INL/EXT-10-18097 Rev. 3, Idaho National Laboratory, 2015.

3. S.B. Grover, D.A. Petti, J.T. Maki, Paper 104, Proceedings of HTR-2010, Prague, Czech Republic, October 18-20, 2010.

4. R.A. Lowden, Fabrication of Baseline and Variant Particle Fuel for AGR-1, ORNL/CF-06/02, Oak Ridge National Laboratory, 2006.

5. J.D. Hunn, T.W. Savage, C.M. Silva, AGR-1 Fuel Compact Pre-Irradiation Characterization Summary Report, ORNL/TM-2012/295, Oak Ridge National Laboratory, 2012.

6. J.A. Phillips, C.M. Barnes, J.D. Hunn, Paper 236, Proceedings of HTR-2010, Prague, Czech Republic, October 18-20, 2010.

7. J.T. Maki, AGR-1 Irradiation Experiment Test Plan, INL/EXT-05-00593 Rev. 3, Idaho National Laboratory, 2009.

8. S.A. Ploger, P.A. Demkowicz, J.D. Hunn, J.S. Kehn, Microscopic analysis of irradiated AGR-1 fuel compacts, Nucl. Eng. Des. 271 (2014) 221-230.

9. S.A. Ploger, P.A. Demkowicz, J.D. Hunn, J.S. Kehn, Ceramographic Examinations of Irradiated AGR-1 Fuel Compacts, INL/EXT-12-25301 Rev. 1, Idaho National Laboratory, 2012.

10. B.P. Collin and S.A. Ploger, AGR1 PIE Measurement of Kernel Swelling and Buffer Densification, and Comparison to Model Predictions by PARFUME, ECAR-2206, Idaho National Laboratory, 2013.

11. P.A. Demkowicz, J.D. Hunn, S.A. Ploger, R.N. Morris, C.A. Baldwin, J.M. Harp, P.L. Winston, T.J. Gerczak, I.J. van Rooyen, F.C. Montgomery, C.M. Silva, Irradiation performance of AGR-1 high temperature reactor fuel, Nucl. Eng. Des. 306 (2016) 2-13.

12. S.R. Eliason, Maximum Likelihood Estimation: Logic and Practice. SAGE, 1993. 
13. J.B.S. Haldane, Moments of the distributions of powers and products of normal variates, Biometrika, 32 (1942) 226-242.

14. J.D. Hunn and R.A. Lowden, Data Compilation for AGR-1 Variant 3 Coated Particle Composite LEU01-49T, ORNL/TM-2006/022, Oak Ridge National Laboratory, 2006.

15. A.K. Kercher, J.D. Hunn, J.R. Price, P.J. Pappano, Automated optical microscopy of coated particle fuel, J. Nucl. Mater. 380 (2008) 76-84.

16. J.D. Hunn. A.K. Kercher, P.A. Menchhofer, and J.R. Price, Results from ORNL Characterization of Nominal $350 \mu \mathrm{m}$ NUCO Kernels from the BWXT 59344 Batch, ORNL/TM-2005/541, Oak Ridge National Laboratory, 2005.

17. L.L.Snead, T. Nozawa, Y. Katoh, T.S. Byun, S. Kondo, D.A. Petti, Handbook of SiC properties for fuel performance modeling, J. Nucl. Mater. 371 (2007) 329-377.

18. G.K. Miller, D.A. Petti, J.T. Maki, D.L. Knudson, PARFUME Theory and Model Basis Report, INL/ EXT-08-14497, Idaho National Laboratory, 2009.

19. L.J. Siefken, E.W. Coryell, E.A. Harvego, J.K. Hohorst, SCDAP/RELAP5/MOD3.3 Code Manual: MATPRO - A Library of Materials Properties for Light-Water-Reactor Accident Analysis, NUREG/ CR-6150, Vol. 4, Rev. 2 (2001).

20. E. Proksch, K. Knotik, A. Strigl, J. Baier, Abbrandbedingtes schwellen hochdichtiger oxidischer HTR-brennstoffkerne, J. Nucl. Mater. 66 (1977) 263-272. 